\title{
Characteristics and rates of mental health problems among Indian and White adolescents in two English cities
}

\author{
Nisha Dogra, Nadzeya Svirydzenka, Pat Dugard, Swaran P. Singh and Panos Vostanis
}

\section{Background}

Sampling techniques for national surveys have constrained the statistical power in estimating prevalence rates of child mental health problems in minority ethnic groups.

\section{Aims \\ To establish the prevalence rates of mental health problems in ethnic Indian adolescents in England and compare these with matched White adolescents living in the same areas. \\ Method \\ A cross-sectional survey with oversampling of Indian adolescents aged 13-15 years of age.}

\section{Results}

The sample size was 2900 (71\% response rate) with 1087 (37\%) Indian and 414 (14\%) White adolescents. Ethnically Indian adolescents had lower rates of all types of mental health problems $(5 \%$ v. $13 \%$ and $21 \%$ v. $30 \%$ for abnormal Strengths and Difficulties Questionnaire and Short Mood and Feelings Questionnaire scores, respectively) and substance misuse $(18 \%$ v. $57 \%, 5 \%$ v. $15 \%$ and $6 \%$ v. $9 \%$ for regular alcohol, smoking and drug use, respectively), with the exception of eating disorders, compared with their White counterparts. The odds of an abnormal score on the mental health questionnaires were worse for White compared with Indian children irrespective of sociodemographic variables.

\section{Conclusions}

Factors relating to how Indian adolescents are parented or their social support networks may be influencing their mental health and may warrant further investigation.

\section{Declaration of interest}

None.
Approximately $20 \%$ of the world's children and adolescents are estimated to have mental health problems that require assessment and intervention, with similar types of psychopathology across cultures. The figure can only be an estimate given that data on child and adolescent mental health is variably reported in national health surveys. ${ }^{1}$ Two surveys in Great Britain established that the point prevalence of mental health problems was $11.5 \%$ among 11 - to 16 -year-olds, $10.3 \%$ among girls and $12.6 \%$ among boys. ${ }^{2}$ Girls had higher rates of emotional problems, and boys had higher rates of conduct and hyperkinetic disorders. These results are similar to the rates found in Germany by the BELLA study, which established that $12.2 \%$ of 11 - to 17 -year-olds met the subclinical or clinical threshold on a standardised morbidity measure $(9.5 \%$ for girls and $14.8 \%$ boys), with gender patterns on the distribution of specific problems similar to the UK population. ${ }^{3}$

Despite the emerging evidence regarding the level of child mental health need in the general population, sampling techniques have constrained the statistical power in estimating similar rates in minority ethnic groups. A trend in the Great Britain survey suggested that, in comparison with White British children, Indian children had lower prevalence of both conduct and emotional problems. ${ }^{2}$ An additional difficulty in interpreting existing data from general population surveys is the way of recording or measuring ethnicity, with relatively heterogeneous groups being grouped together. ${ }^{4}$

The increasing diversity of society and the importance of developing services based on clear evidence require detailed knowledge about the specific needs of different ethnic groups. This was the rationale for the present study. Its objectives were: (a) to establish the prevalence rates of mental health problems in settled communities of ethnic Indian adolescents in England; (b) to compare these rates with those of matched White adolescents living in the same areas; and (c) to determine sociodemographic correlates of any differences found. In this paper we use the term Indian and White adolescents as identified by their ethnicity (by ethnicity we mean their background heritage and origins), not nationality. Indian adolescents include those with British, Indian and other nationalities, and White adolescents include other nationalities as well as British.

\section{Method}

\section{Recruitment and sample}

We deliberately sought to oversample Indian adolescents to obtain a large enough group for reliable estimation of prevalence rates. For this, we stratified our sample by Indian ethnicity, the majority minority ethnic population in Leicester and West London based on Census data, ${ }^{5-7}$ and approached schools that had significant numbers of adolescents (over 50\%) of Indian ethnicity. There were no other inclusion criteria, although all the schools fulfilling the first criterion were in urban areas. The age group 13-15 years was selected because of the increase of mental health problems in adolescence, ${ }^{2}$ and the schools' suggestion not to involve pupils during their final examinations year.

\section{Materials}

All adolescents completed the following instruments.

\section{Sociodemographic checklist}

This included information on age, gender, young person's and parents' place of birth, religion, primary language and any other languages they spoke, nationality, whether they felt that they belonged to an ethnic group (and if so, were given a list to select from), whether they perceived they belonged to one culture or more (and to identify these), who they lived with, and whether they qualified for free school meals (as a measure of socioeconomic 
status). Ethnicity was not defined and the list used contained similar groups as used in the census.

\section{Mental health assessment instruments}

These were widely established and standardised measures of different types of mental health problems that are particularly common in adolescence.

(a) The Strengths and Difficulties Questionnaire (SDQ), ${ }^{8}$ as a measure of general mental health problems, with a score of total problems, as well as subscales on conduct, emotional, hyperkinetic and peer relationships problems.

(b) The Short Mood and Feelings Questionnaire (SMFQ), ${ }^{9}$ a measure of depressive symptoms.

(c) The SCOFF (abridged UK version), ${ }^{10}$ a measure of eating problems.

Questions were also asked on the use of drugs, alcohol and smoking. The questionnaire and supporting materials were piloted on a class of 24 adolescents of various ethnicities from one of the schools recruited for clarity and comprehension, which resulted in minor alterations to the language and procedure.

\section{Research procedure}

As the study did not involve patients, the project was approved by the University of Leicester Ethics Committee. Data collection took place between September 2010 and May 2011. The procedures varied slightly from school to school to fit in with the school's schedules. Each school received training from a researcher on how the questionnaires were to be administered. Following communication with their parents on the aims of the study, pupils were provided with an outline of the project and an opportunity to consider their participation. If they provided informed consent, they completed the measures, which were sealed and returned to the teacher.

\section{Statistical analysis}

Questionnaires were coded and entered onto SPSS 18 for Windows. The mental health questionnaires were dichotomised according to previously established cut-off scores that distinguished clinical from non-clinical cases, i.e. children who were likely to benefit from assessment and possible intervention. Scores of $0-5$, or 6 , or $7-10$ for the emotional and hyperactivity SDQ subscales led to normal, borderline and abnormal categories respectively for these subscales. For the conduct subscale, scores of 0-3, 4 and 5-10 led to categorisation as normal, borderline and abnormal respectively. For peer problems, scores of $0-3$ are considered normal, $4-5$ borderline and 6-10 abnormal. The usual practice is that prosocial scores are scored separately and not included in the total SDQ and we followed that convention. A score of $6-10$ is normal, 5 is borderline and any score lower than 4 abnormal. Total SDQ scores vary from 0 to 40 with $0-15,16-19$ and 20-40 falling in normal, borderline and abnormal ranges for developing or having mental health problems respectively. An SMFQ score of eight and above showed that the young person was at risk of depression or anxiety problems. Those children with scores of 3 and higher on a $0-5$ SCOFF score range are at risk of having or developing an eating disorder. Chi-squared tests were completed for between-group comparisons.

Regression is often the method of choice for attempting to predict a variable that may be influenced by several other variables. It was not appropriate for our analysis as we were trying to predict categories (normal, borderline or abnormal) for our mental health status variables SDQ and SMFQ. The variables we expect to be associated with mental health status are also categorical: gender, whether they received free school meals, whether they see themselves as having a single cultural identity, who they live with, ethnic group. An extension to several variables of the chi-squared test of association in a two-way table is required and this is provided by a log-linear model. ${ }^{11,12}$

A two-way table has only two possibilities: the row and column variables are independent, or they are associated. If there are three variables, then the row, column and layer variables may all be independent, or the row and column variables may be independent within each layer but the layer variable is associated with the row and the column variables, or there may be a threeway association, or there are other possibilities. Adding variables rapidly increases the possibilities. Log-linear models provide a general framework for dealing with such problems hence our justification for using these, rather than regression, models.

\section{Results}

Of 23 schools contacted with preliminary information about the project, 9 (5 in Leicester of which one was a girls-only school and 4 in London) participated, giving a response rate of $39 \%$. The sample comprised of 2900 adolescents from a possible 4082 giving a response rate of $71 \%$. The mean age was 13.97 years (s.d.=0.7). As we surveyed school years 9 and 10, most of our sample was aged between 13 and 15 years $(734(25.3 \%)$ were 13 ; $1500(51.7 \%)$ were $14 ; 636(21.9 \%)$ were 15 and $6(0.2 \%)$ were 16). Twenty-four $(0.8 \%)$ did not provide information about their age. There were 1284 (44\%) boys and 1610 (56\%) girls (6 individuals did not provide information on gender). There were 1087 (38\%) Indian and $414(14 \%)$ White adolescents and it is these two groups we will be focusing on in the analysis. In the Indian sample there were 490 males (45.1\%) and 597 females (54.9\%). In the White British sample 148 (35.7\%) were male and 266 $(64.3 \%)$ were female. The mean age of the Indian group was 13.91 (s.d. =0.68) and that of the White group 14.05 (s.d.=0.7) years and for this reason age is not considered further. The analysis does not include White Other and focuses only on those who identified themselves as White British to reduce heterogeneity.

\section{Relationship between sociodemographic factors and ethnicity}

Online Table DS1 summarises the results of comparisons on demographic factors (for some variables the categories have been combined: for example, there are many main languages other than English and many nationalities other than British, but numbers are often in single figures and we have grouped them as shown in online Table DS1). For the family situation factor, the category 'lives with both parents' includes those living with their extended family, as there were almost identical proportions in the abnormal or borderline ranges of the mental health scales for participants in two-parent and extended families. Many of the results of these comparisons are expected: higher proportions of Indian than White adolescents have a main language other than English $\left(\chi^{2}(1)=23.16, P<0.001\right)$ and a nationality other than British $\left(\chi^{2}(2)=95.85, P<0.001\right)$. There was little overlap in religious group affiliations between Indian (57\% Hindu, 27\% Muslim) and White British adolescents (42\% Christian, $40 \%$ agnostic/atheist). Similar proportions of the groups qualify for free school meals. Less than half of Indian but three-quarters of White adolescents' state they have a single cultural identity $\left(\chi^{2}(1)=61.19, P<0.001\right)$. A much higher proportion of Indian than White adolescents live with both 
parents or their extended family, and correspondingly fewer with a single-parent or stepfamily $\left(\chi^{2}(2)=124.50, P<0.001\right)$.

\section{Ethnicity (self-identified) and reported mental health problems}

Indian adolescents reported lower proportions in the abnormal and borderline SDQ range (Table 1), indicating lower rates of general mental health (behavioural and emotional) problems. A lower proportion of the Indian sample (21\%) had scores in the abnormal SMFQ range (depressive symptoms) than the White sample (30\%). There were no differences between the two groups on SCOFF relating to eating problems. A higher proportion of White adolescents tried alcohol and drugs and then went on to use them regularly $(57 \% v .18 \%)$. The differences were greatest for alcohol, but were also significant for smoking and illicit drugs.

As the girls-only school had a high proportion of White adolescents, the White sample had a higher proportion of girls (64\%) than the Indian sample (55\%). Rates were therefore compared by ethnicity and gender (Tables 2 and 3). White boys had significantly higher rates of borderline or abnormal SDQ total scores and were significantly more likely to use alcohol than Indian boys. For all measures, except the SCOFF, White girls had higher rates of problems and substance misuse than Indian girls. Hence, the White sample having more girls does not explain the rates for White children being higher.

\section{Gender characteristics among Indian adolescents}

Within the Indian group, boys had significantly higher proportions of abnormal and borderline scores $\left(\chi^{2}(2)=6.92, P<005\right)$ than girls on the SDQ conduct subscale, but significantly lower proportions of abnormal and borderline scores on the emotional subscale $\left(\chi^{2}(2)=29.31, P<0001\right)$. There were no statistically significant differences between the two groups on the SDQ hyperkinetic or peer relationships subscales, or the SCOFF. The girls' higher SDQ emotional subscale scores were consistent with girls showing significantly higher rates on the SMFQ $\left(\chi^{2}(1)=\right.$ 19.25, $P<0001)$ than boys. Indian boys were significantly more likely to smoke $\left(\chi^{2}(2)=14.14, P<0.01\right)$ and use illicit drugs $\left(\chi^{2}(2)=26.82, P<0.001\right)$ compared with their Indian female peers.

\section{Relationship between sociodemographic factors and mental health problems}

Age was not considered as a variable as all but one of our sample was aged between 13 and 15 years (all were from school years 9 and 10). Comparing Indian and White participants who say they identify with a single culture reveals that a significantly lower proportion of Indian adolescents were in the abnormal or borderline range of SDQ total $\left(\chi^{2}(2)=11.95, P<0.01\right)$ and SMFQ scores $\left(\chi^{2}(1)=5.98, P<0.05\right)$. Similar results are obtained when Indian and White adolescents who do not perceive that they

\begin{tabular}{|c|c|c|c|c|}
\hline & \multicolumn{2}{|c|}{ Ethnic group, $n(\%)$} & \multirow[b]{2}{*}{$\chi^{2}$ (d.f.) } & \multirow[b]{2}{*}{$P$} \\
\hline & Indian & White & & \\
\hline \multicolumn{5}{|c|}{ scale and score range } \\
\hline \multicolumn{5}{|c|}{ Strengths and Difficulties Questionnaire total } \\
\hline Normal & $797(82)$ & $255(70)$ & & \\
\hline Borderline & $125(13)$ & $63(17)$ & & \\
\hline Abnormal & $48(5)$ & $46(13)$ & & \\
\hline Total & $970(100)$ & $364(100)$ & $3080(2)$ & $<0.001$ \\
\hline \multicolumn{5}{|c|}{ Strengths and Difficulties Questionnaire prosocial } \\
\hline Normal & $884(84)$ & $312(78)$ & & \\
\hline Borderline & $104(10)$ & $59(15)$ & & \\
\hline Abnormal & $58(6)$ & $27(7)$ & & \\
\hline Total & $1046(100)$ & $398(100)$ & $814(2)$ & $<0.05$ \\
\hline \multicolumn{5}{|c|}{ Short Mood and Feelings Questionnaire total } \\
\hline Normal & $818(79)$ & $276(70)$ & & \\
\hline Abnormal & $221(21)$ & $116(30)$ & & \\
\hline Total & $1039(100)$ & $392(100)$ & 1095 (1) & $<0.01$ \\
\hline \multicolumn{5}{|l|}{ SCOFF total } \\
\hline Normal & $927(92)$ & $356(92)$ & & \\
\hline Abnormal & $81(8)$ & $31(8)$ & & \\
\hline Total & $1008(100)$ & $387(100)$ & $0(1)$ & 1 \\
\hline \multicolumn{5}{|c|}{ Substance, usage } \\
\hline \multicolumn{5}{|c|}{ Drugs } \\
\hline Never & $940(90)$ & $341(85)$ & & \\
\hline Once only & $46(4)$ & $26(6)$ & & \\
\hline Regular & $63(6)$ & $35(9)$ & & \\
\hline Total & $1049(100)$ & $402(100)$ & 643 & $<0.05$ \\
\hline \multicolumn{5}{|l|}{ Alcohol } \\
\hline Never & 709 (68) & 108 (27) & & \\
\hline Once only & 148 (14) & $67(17)$ & & \\
\hline Regular & $190(18)$ & 229 (57) & & \\
\hline Total & 1047 (100) & $404(100)$ & 23806 & $<0.001$ \\
\hline \multicolumn{5}{|l|}{ Smoking } \\
\hline Never & $892(85)$ & $268(66)$ & & \\
\hline Once only & $105(10)$ & 75 (19) & & \\
\hline Regular & $55(5)$ & $61(15)$ & & \\
\hline Total & $1052(100)$ & $404(100)$ & 6557 & $<0.001$ \\
\hline
\end{tabular}




\begin{tabular}{|c|c|c|c|c|}
\hline & \multicolumn{2}{|c|}{ Ethnic group, $n(\%)$} & \multirow[b]{2}{*}{$\chi^{2}$ (d.f.) } & \multirow[b]{2}{*}{$P$} \\
\hline & Indian & White & & \\
\hline \multicolumn{5}{|c|}{ Scale and score range } \\
\hline \multicolumn{5}{|c|}{ Strengths and Difficulties Questionnaire total } \\
\hline Normal & $362(84)$ & $101(75)$ & & \\
\hline Borderline & $49(11)$ & $19(14)$ & & \\
\hline Abnormal & $21(5)$ & $14(11)$ & & \\
\hline Total & $432(100)$ & $134(100)$ & $6.73(2)$ & $<0.05$ \\
\hline \multicolumn{5}{|c|}{ Strengths and Difficulties Questionnaire prosocial } \\
\hline Normal & $356(76)$ & $100(70)$ & & \\
\hline Borderline & $73(16)$ & $28(20)$ & & \\
\hline Abnormal & $38(8)$ & $15(10)$ & & \\
\hline \multirow{2}{*}{\multicolumn{5}{|c|}{ Short Mood and Feelings Questionnaire total }} \\
\hline & & & & \\
\hline Normal & $402(85)$ & $111(79)$ & & \\
\hline Abnormal & 72 (15) & 29 (21) & & \\
\hline Total & $474(100)$ & $140(100)$ & $2.40(1)$ & $>0.05$ \\
\hline \multicolumn{5}{|l|}{ SCOFF total } \\
\hline Normal & $429(93)$ & $132(96)$ & & \\
\hline Abnormal & $30(7)$ & $5(4)$ & & \\
\hline Total & $459(100)$ & $137(100)$ & $1.59(1)$ & $>0.05$ \\
\hline \multicolumn{5}{|c|}{ Substance, usage } \\
\hline \multicolumn{5}{|l|}{ Drugs } \\
\hline Never & $401(85)$ & $126(86)$ & & \\
\hline Once only & $26(5)$ & $9(6)$ & & \\
\hline Regular & $47(10)$ & $11(8)$ & & \\
\hline Total & $474(100)$ & $146(100)$ & $0.81(2)$ & $>0.05$ \\
\hline \multicolumn{5}{|l|}{ Alcohol } \\
\hline Never & $321(68)$ & $39(27)$ & & \\
\hline Once only & 70 (15) & $26(18)$ & & \\
\hline Regular & $81(17)$ & $81(55)$ & & \\
\hline Total & $472(100)$ & $146(100)$ & $95.74(2)$ & $<0.001$ \\
\hline \multicolumn{5}{|l|}{ Smoking } \\
\hline Never & $381(80)$ & $111(76)$ & & \\
\hline Once only & $61(13)$ & $22(15)$ & & \\
\hline Regular & $33(7)$ & $13(9)$ & & \\
\hline Total & $475(100)$ & $146(100)$ & $1.24(2)$ & $>0.05$ \\
\hline
\end{tabular}

belong to only one cultural group are analysed, although proportions differ significantly only for the SDQ total scores $\left(\chi^{2}(2)=16.88, P<0.001\right)$.

Comparing Indian and White adolescents who identified English as their main language, there is the same pattern of lower proportions of Indian adolescents in the borderline or abnormal ranges of the SDQ total and the SMFQ, and using drugs, alcohol and tobacco as when we compare the full Indian and White samples, as the comparison is in effect the same given the majority had English as their main language. Again the proportions with abnormal SCOFF scores are similar for the two ethnic groups. The small numbers of those whose main language is not English means that chi-squared tests are not appropriate, but the same pattern can be seen in all the tables as we observed for those with English as the main language, or indeed for the ethnic groups as a whole. Within the British nationality group we find higher proportions of White than Indian adolescents in the abnormal or borderline ranges of SDQ total and SMFQ scores. Also, White adolescents had higher prevalence for regular alcohol use compared with Indian adolescents (58\% and $16 \%$ respectively). For smoking the figures are $14 \%$ and $5 \%$, and for drug use $8 \%$ and $7 \%$ (the difference in proportions is not significant for the latter).

Similarly, among those who qualified for free school meals, White adolescents showed higher abnormal ranges of SDQ total and SMFQ scores than their Indian counterparts. A higher proportion of White adolescents also exhibited regular alcohol consumption and smoking behaviour. Among those not qualifying for free school meals we found the same results except that this time significantly more White adolescents also used drugs.

Those living with either both parents or extended family have lower proportions in the abnormal or borderline ranges of the SDQ total, SMFQ and SCOFF than those living with a single parent, who in turn have lower proportions than those in stepfamilies. All these differences are significant at the $1 \%$ level and are considerable. Combining abnormal and borderline ranges to summarise SDQ total, the percentages are $18 \%, 28 \%$ and $42 \%$ for SDQ total, $21 \%, 29 \%$ and $44 \%$ for SMFQ, $7 \%, 11 \%$, and $17 \%$ for SCOFF. There are also significant differences in proportions using drugs, alcohol and tobacco, with those in two-parent or extended families again at a clear advantage and those in stepfamilies with the highest rates of use.

The five variables that may be associated with mental health status are all binary except for who children live with. In this case, as there were only 73 living with stepfamilies, these were combined with single-parent families giving two categories: lives with both parents or extended family, lives with a single parent or stepfamily. This led to five binary variables, so 32 cells in a five dimensional table. 


\begin{tabular}{|c|c|c|c|c|}
\hline & \multicolumn{2}{|c|}{ Ethnic group, $n(\%)$} & \multirow[b]{2}{*}{$\chi^{2}$ (d.f.) } & \multirow[b]{2}{*}{$P$} \\
\hline & Indian & White & & \\
\hline \multicolumn{5}{|c|}{ scale and score range } \\
\hline \multicolumn{5}{|c|}{ Strengths and Difficulties Questionnaire total } \\
\hline Normal & $435(81)$ & $154(67)$ & & \\
\hline Borderline & $76(14)$ & $44(19)$ & & \\
\hline Abnormal & $27(5)$ & $32(14)$ & & \\
\hline Total & $538(100)$ & $230(100)$ & $23.23(2)$ & $<0.001$ \\
\hline \multicolumn{5}{|c|}{ Strengths and Difficulties Questionnaire prosocial } \\
\hline Normal & $528(91)$ & $212(83)$ & & \\
\hline Borderline & $31(5)$ & $31(12)$ & & \\
\hline Abnormal & $20(4)$ & $12(5)$ & & \\
\hline Total & $579(100)$ & $255(100)$ & 13.04 (2) & $<0.01$ \\
\hline \multicolumn{5}{|c|}{ Short Mood and Feelings Questionnaire total } \\
\hline Normal & $416(74)$ & $165(65)$ & & \\
\hline Abnormal & $149(26)$ & $87(35)$ & & \\
\hline Total & $565(100)$ & $252(100)$ & $5.63(1)$ & $<0.05$ \\
\hline \multicolumn{5}{|l|}{ SCOFF total } \\
\hline Normal & 498 (91) & $224(90)$ & & \\
\hline Abnormal & $51(9)$ & $26(10)$ & & \\
\hline Total & $549(100)$ & $250(100)$ & $0.24(1)$ & $>0.05$ \\
\hline \multicolumn{5}{|c|}{ Substance, usage } \\
\hline \multicolumn{5}{|l|}{ Drugs } \\
\hline Never & 539 (94) & $215(84)$ & & \\
\hline Once only & 20 (3) & $17(7)$ & & \\
\hline Regular & $16(3)$ & $24(9)$ & & \\
\hline Total & $575(100)$ & $256(100)$ & $21.83(2)$ & $<0.001$ \\
\hline \multicolumn{5}{|l|}{ Alcohol } \\
\hline Never & 388 (67) & $69(27)$ & & \\
\hline Once only & $78(14)$ & $41(16)$ & & \\
\hline Regular & 109 (19) & $148(57)$ & & \\
\hline Total & 575 (100) & 258 (100) & 139.69 (2) & $<0.001$ \\
\hline \multicolumn{5}{|l|}{ Smoking } \\
\hline Never & $511(87)$ & 157 (61) & & \\
\hline Once only & $44(8)$ & $53(21)$ & & \\
\hline Regular & $22(4)$ & $48(19)$ & & \\
\hline Total & 577 (100) & $258(100)$ & 87.25 (2) & $<0.001$ \\
\hline
\end{tabular}

The SDQ and SMFQ indicate mental health status. The next step examines how the variables discussed above are associated with mental health status, which in binary terms can be viewed as no abnormal scores on the SDQ or SMFQ or at least one abnormal score, thus giving a summary mental health indicator. In total there are six variables, which gives a six-way table to see how the variables relate to each other. As with a two-way table and the chi-squared test, we were interested in the interactions or associations in our six-way table. As SPSS provides for this facility, backward elimination was used to identify the interactions that were significant. (There are two goodness-of-fit tests, Pearson and likelihood ratio, which indicate a poor fit if they reach significance: our significance levels are 0.86 and 0.72 respectively indicating that the model is effective.) The model presented includes all interactions among gender, ethnic group, who the young person lives with and whether they have a single cultural identity. In addition, it includes all interactions among school meals, who they live with and the mental health indicator, and the interactions between the mental health indicator and gender, and between the mental health indicator and ethnic group. After allowing for all other interactions, there is still an association between mental health indicator and ethnic group that is significant at the $5 \%$ level $(P=0.016)$.

The log-linear model provides expected cell counts for each of the 64 cells in the six-way table: these can be seen with observed cell counts in online Table DS2. For any combination of variables (such as male, White, free school meals, single cultural identity, lives with both parents or extended family), the odds of an abnormal score on the mental health indicator is the ratio of expected numbers in this combination of categories with and without an abnormal score on the mental health indicator (1.313/4.031 or 0.3257 for this example). Using the same example categories except for Indian replacing White, the ratio of expected numbers of male, Indian, free school meals, single cultural identity, lives with both parents or extended family, with and without an abnormal score as the mental health indicator, $3.208 / 14.363$ or 0.2234 . The odds are worse for the White group (0.3257) than for the Indian adolescents (0.2234) who are otherwise similar. In fact, the odds ratio White/Indian is 1.46. This is the case for all other combinations of variables: the odds of an abnormal score on the mental health indicator are worse for White than for Indian children and the odds ratio of 1.46 gives an estimate of the size of the effect of ethnic group after allowing for our other variables.

\section{Discussion}

\section{Main findings}

This study found that adolescents living in England and who were ethnically Indian had lower rates of all types of mental health 
problems and substance misuse than White adolescents. This adds to previous findings that Indian children are predominantly protected against externalising problems. ${ }^{13}$ The rates also reflect the patterns of mental health problems among indigenous adolescents of a similar age in India. ${ }^{14}$ Although the proportion of problems and their gender associations were similar to the White population (higher rates of emotional problems for girls and higher rates of conduct problems for boys), as established by other studies, ${ }^{2,3,15}$ the rates were significantly lower for all problems apart from eating disorders. The rate of possible eating disorders in our study was $8 \%$ and this is considerably less than that identified in a German study of 1895 young people aged between 11 and 17 years, which found that $29 \%$ of girls and $14 \%$ of boys scored beyond the normal threshold. ${ }^{15}$ The higher use of substances in boys compared with girls, found in the Indian and White groups, reflects the same pattern as identified by previous research. ${ }^{2,3}$

Comparing Indian and White adolescents who perceived they belonged to only one cultural group, the former scored lower for some problems, and it may be that in these areas where there is high presence of Indians or other ethnic groups, White adolescents are the 'minority' group who experience more marginalisation resulting in their higher rates of problems. Georgiades et al ${ }^{16}$ found that, despite greater socioeconomic disadvantage, young people living in recent immigrant families had lower levels of emotional/behavioural problems and higher levels of school performance. Living in a neighbourhood with higher concentration of immigrants was associated with lower levels of problems among immigrant children but the reverse was true for non-immigrant children. Living in high-immigrant areas as an immigrant may provide community support whereas in White groups it may indicate socioeconomic disadvantage. This in part may explain some of the differences found, although most of the adolescents surveyed are unlikely to be 'immigrants' in that most were UK born. The high rate of multiple culture identification in Indian adolescents who also score well on mental health instruments suggests that cultural identification might have a mediating role in mental health problem prevalence, but this question requires targeted hypothesis-driven research.

As in previous research, adolescents in two-parent families are at lower risk of mental health problems than those from loneparent or reconstituted families. ${ }^{2}$ With the large differences in proportions of Indian and White adolescents in the different family situations, and the strong association between family situation and proportions in abnormal or borderline ranges of the mental health scales and proportions using drugs, alcohol and tobacco, the question is whether the advantage in family situation of the Indian adolescents explains their mental health advantage. However, this was not supported by the finding that when considering only those in two-parent or extended families the Indian adolescents have significantly lower proportions in the abnormal or borderline range of the SDQ total score (the SMFQ score is also lower but does not reach significance). The pattern is similar for single-parent families. Although divorce rates in the British Indian population may be increasing, ${ }^{17}$ they are still the lowest at $10 \% .^{5-7}$ It may be that Indian adolescents are less likely to have to adjust to reconstituted families, and may also receive greater support from extended family networks, thereby mitigating the impact of parental separation.

Religion as a protective and risk factor has also been studied, ${ }^{18}$ but in our sample belonging to a specific religious group appeared not to be a significant factor either way, although we did not measure how religious adolescents were in practice. Poverty is another well-established risk factor for child mental health problems. In this study we used the index entitlement to free school meals, which seems to have only a small effect but still, within those groups who qualify for free school meals and those who do not, Indian adolescents remain at an advantage. It does not appear that the two ethnic groups have different levels of poverty so that does not help explain the difference in the rates of problems identified. The study took place in the city of Leicester and in Wembley, neither of which are overtly affluent areas. The issue of socioeconomic disadvantage may be mitigated if children do not feel worse off than their peers because the level of disadvantage is possibly equal across the group.

\section{Implications}

Overall, adolescents of Indian ethnic background appear to enjoy better mental health than their White counterparts. Our data show the difference but do not explain it. There may be factors relating to how children are parented or their social support networks that are influencing their mental health that may warrant further investigation. This study has also demonstrated that national data samples may be of less use for services that do not reflect the national picture and we would suggest that repeating such surveys in areas with high minority populations may be useful. More in-depth research about the role of potentially mediating factors such as religion, culture and ethnicity is important before drawing definitive conclusions.

\section{Limitations}

A number of limitations need to be acknowledged. The study relies on self-report questionnaires, which are less accurate than diagnostic interviews. However, the components comprising the questionnaire have all been well validated and the same process was used to collect data in both groups. Those adolescents with mental health problems may have chosen to absent themselves from school when they were expected to complete the questionnaires but again this would apply to both ethnic groups. Although the measures used are well validated, they have their own limitations given that they are all self-report measures. The SDQ, for example has only moderate sensitivity of around 15\% for all disorders. ${ }^{19}$ Also, the SDQ has been validated for use in British samples, most of which have been White. ${ }^{2}$ In the Office for National Statistics survey on child mental health in which the SDQ was used, there were no comments or reports (Indian sample size 361) that the cut-offs for this ethnic group needed to be different. We are not aware of any studies in 'Indian ethnicity' children that have used the SDQ and then a goldstandard psychiatric interview, so the SDQ has not been calibrated for Indian children and it is possible that a different cut-off for risk of disorder would be needed. An additional limitation is that there were not multiple informants and SDQs, when completed by parents and teachers, are generally better predictors than SDQs completed just by the adolescent. ${ }^{20}$ There is also potential inconsistency in how the questionnaires were administered across different schools, although we mitigated this by providing clear guidance and research staff to help with the process if needed. There was no corroborative information from parents, or more detailed measurement of identity factors and other potential mediating variables, such as parenting attitudes and practice. There were no major differences between those schools that participated and those that did not, although those that did may have had staff with more awareness of mental health issues.

\section{Future research}

In addition to addressing these issues, future research could replicate this study with other ethnic groups. This would establish 
the patterns of mental health problems across different communities, to enhance the understanding of the social mechanisms that might account for the variations that exist between ethnic groups, but are accounted for by more factors than ethnicity per se. It would also inform reliable health services recording and service planning based on evidence of children's and young people's mental health needs.

Nisha Dogra, PhD, Nadzeya Svirydzenka, PhD, Greenwood Institute of Child Health, School of Psychology, University of Leicester; Pat Dugard, MA, School of Psychology, University of Dundee; Swaran P. Singh, DM, Mental Health \& Wellbeing University of Warwick, Coventry; Panos Vostanis, MD, Greenwood Institute of Child Health, School of Psychology, University of Leicester, UK

Correspondence: Dr N. Dogra, Greenwood Institute of Child Health, Schoo of Psychology, University of Leicester, Westcotes House, Westcotes Drive, Leicester LE3 0QU, UK. Email: nd13@|e.ac.uk

First received 16 Jan 2013, final revision 21 Mar 2013, accepted 3 Apr 2013

\section{Funding}

The National Institute for Health Research (NIHR) Clinical Research Networks funded the project.

\section{Acknowledgements}

We are grateful to all the young people who took part in this study. We also thank the parents and teachers for their kind cooperation, and Dr David Clarke Associate Director of the Leicestershire Partnership NHS Trust, for his ongoing support.

\section{References}

1 World Health Organization. Atlas on Child and Adolescent Mental Health Resources - Global Concerns: Implications for the Future. World Health Organization, 2005

2 Green H, McGinnity A, Meltzer H, Ford T, Goodman R. Mental Health of Children and Young People in Great Britain, 2004. Office for National Statistics, 2005

3 Ravens-Sieberer $\mathrm{U}$, Wille $\mathrm{N}$, Erhart $\mathrm{M}$, Bettge $\mathrm{S}$, Wittchen $\mathrm{H}$, Rothenberger $\mathrm{A}$ et al. Prevalence of mental health problems among children and adolescents in Germany: results of the BELLA study within the National Health Interview and Examination survey. Eur Child Adolesc Psychiatry 2008; 17 (suppl 1): 22-33.
4 Dogra N, Singh SP, Svirydzenka N, Vostanis P. Mental health problems in children and young people from minority ethnic groups: the need for targeted research. Br J Psychiatry 2012; 200; 265-7.

5 Leicester City Council. Census 2001. Office for National Statistics, 2003 (http://www.leicester.gov.uk/your-council-services/council-and-democracy/ city-statistics/census2001)

6 Brent Council. Brent Borough Census 2001 (https://intelligence.brent.gov.uk/ Pages/default.aspx).

7 Harrow Council. Harrow Borough Census 2001 (http://www.harrow.gov.uk/ info/200088/statistics_and_census_information/658/census_information/8).

8 Goodman R (2001). Psychometric properties of the Strengths and Difficulties Questionnaire (SDQ). J Am Acad Child Adolesc Psychiatry 2001; 40: 1337-45.

9 Angold A, Costello EJ, Messer SC, Pickles A, Winder F, Silver D. The development of a short questionnaire for use in epidemiological studies of depression in children and adolescents. Int J Methods Psychiatr Res 1995; 5: 237-49.

10 Morgan JF, Reid F, Lacey JH. The SCOFF questionnaire: assessment of a new screening tool for eating disorders. BMJ 1999; 319: 1467-68.

11 Dugard P, Todman J, Staines H. Approaching Multivariate Analysis, 2nd edn. Routledge, 2010.

12 Howell DC. Statistical Methods for Psychology, 7th edn. Wadsworth, 2010.

13 Goodman A, Patel V, Leon D. Why do British Indian children have an apparent mental health advantage? J Child Psychol Psychiatry 2010; 51: 1171-83.

14 Pillai A, Patel V, Cardozo P, Goodman R, Weiss HA, Andrew G. Non-traditional lifestyles and prevalence of mental disorders in adolescents in Goa, India. Br J Psychiatry 2008; 192: 45-51.

15 Herpertz-Dahlmann $B$, Wille $\mathrm{N}$, Holling $\mathrm{H}$, Vloet $\mathrm{T}$, Ravens-Sieberer $\mathrm{U}$, BELLA study group. Disordered eating behaviour and attitudes, associated psychopathology and health related quality of life: results of the BELLA study. Eur Child Adolesc Psychiatry 2008; 17 (suppl 1): 82-91.

16 Georgiades K, Boyle MH, Duku E. Contextual influences on children's mental health and school performance: the moderating effects of family immigrant status. Child Dev 2007; 78: 1572-91.

17 Das C. Resilience, risk and protective factors for British-Indian Children of Divorce. J Soc Sci 2010; 25: 97-108.

18 Meltzer H, Dogra N, Vostanis $\mathrm{P}$, Ford T. Religiosity and the mental health of adolescents in Great Britain. Ment Health Relig Cult 2011; 14: 703-13.

19 Goodman R, Renfrew D, Mullick M. Predicting type of psychiatric disorder from Strengths and Difficulties Questionnaires (SDQ) scores in child mental health clinics in London and Dhaka. Eur Child Adolesc Psychiatry 2000; 9: $129-34$.

20 Goodman R, Ford T, Simmons H, Gatward R, Meltzer H. Using the Strengths and Difficulties Questionnaire (SDQ) to screen for child psychiatric disorders in a community sample. Br J Psychiatry 2000; 177: 534-9. 